\title{
A implementação de Jogos de Computador Educacionais: uma visão geral.
}

Leandro Augusto Kisielewicz

André Koscianski

\begin{abstract}
Resumo
O espectro de possibilidades para relacionar a sala de aula com tecnologias de informação e comunicação é muito amplo; dentro dele, uma linha particular de investigação trata da adaptação ou desenvolvimento de jogos com propósitos didáticos. Esse tema foi visionado há três ou quatro décadas e tornouse um item recorrente na agenda atual de pesquisadores em ensino. Explorar as características dos jogos como fator de motivação ou como veículo ou facilitador de aprendizagem são temas ricos de pesquisa.

Os indicadores positivos do potencial como recurso pedagógico não são, infelizmente, acompanhados de investimentos em jogos educacionais semelhantes aos existentes nos jogos comerciais. Assim há uma tendência de encontrar softwares voltados para ensino pouco interessantes ou com menor qualidade de acabamento gráfico e artístico. Do ponto de vista pedagógico, também é possível detectar deficiências que podem ser fruto de um mal planejamento ou de superficialidade na concepção dos aplicativos.

Para compreender melhor o problema, apresenta-se aqui uma visão geral sobre o assunto. Discutem-se jogos eletrônicos enquanto ferramentas de ensino e são ponderados critérios para a criação de softwares desse tipo.

Palavras-chave: jogos, educação, engajamento, motivação.

\section{Abstract \\ Quality Criteria for Serious Game}

Among the broad range of possibilities relating the classroom and information technologies, a very particular subject is that of developing videogames with educational purposes. This theme was envisioned more than three decades ago and has become a recurring item on current education researchers schedule. Exploring the engaging and motivational characteristics of games with the goal of motivating students and facilitating learning is a rich subject of research.
\end{abstract}


However, the indications in favor of educational games generally do not seem enough to bring investments to the sector. This way there is a tendency for educational games to have poor interfaces and low attractivity. From a pedagogical viewpoint it is also possible to find software with defficiencies that can be the result of insufficient planning or a shallow approach to the project.

We present an overview of the subject. Games are discussed as a teaching tool, in order to consider criteria for the creation of such educational software.

Keywords: games, education, engagement, motivation.

\section{Introdução}

Manter os alunos engajados é, ou deveria ser, uma das principais metas dos professores. 0 engajamento é um fator crucial para que os alunos aprendam e retenham melhor (Vrasidas, 2004).

Obter esse efeito, no entanto, parece tornar-se cada vez mais um desafio em função do cotidiano dos estudantes. Celulares, televisão, computadores e internet competem entre si, oferecendo conteúdos especialmente concebidos para atrair a atenção. Comparativamente, o professor precisa ter muita habilidade para manter o foco de uma classe frente ao antigo quadro de giz. Essa mudança de forma de apresentação é evidente se, por exemplo, compararmos livros didáticos atuais com os livros da década de 70. As mudanças, vale frisar, não são apenas cosméticas. Embora de maneira geral exista um apelo visual mais acentuado em materiais didáticos, seria apressado concluir que o que está em questão é tão somente o aspecto estético. Diversos estudos sinalizam modificações em relação à comunicação, hábitos de vida e na forma de pesquisar e trabalhar com informações: "today's students think and process information fundamentally differently from their predecessors" ${ }^{1}$ (Prensky, 2001a)

Essas modificações passam pela apresentação, pela linguagem, pelo uso cotidiano de ferramentas como celular, e-mail ou Tweeter. É legítimo aventar que, inexoravelmente, a escola irá incorporar tecnologias de informação e comunicação com intensidade crescente. Em países desenvolvidos a disponibilidade de recursos e a receptividade cultural tendem a facilitar esse processo (Badilla-Saxe, 2004); no Brasil subsiste ainda a discussão entre a possibilidade de sucesso contra os resultados efetivamente obtidos. Um motivo para persistir essa conotação de "novas tecnologias" é o custo das ferramentas, que limita ou restringe sua divulgação. Paralelamente a essa realidade na educação, os jogos de computador representam um mercado em franca

\footnotetext{
${ }^{1}$ Os estudantes hoje pensam e processam informação fundamentalmente diferente de seus predecessores.
} 
expansão no país. Além de empresas estrangeiras que trazem títulos em português e começam a instalar escritórios aqui, observa-se a criação de fabricantes nacionais de jogos.

Existe uma rica variedade de gêneros de videogames, que podem ser moldados para diferentes situações de ensino-aprendizagem. Isto permite abrigar desde atividades de verificação de conhecimento ou memorização, até percursos relativamente livres de exploração de material e teste de hipóteses. Quando concebidos em bases epistemológicas corretas, os jogos podem efetivamente funcionar como meio de aprendizagem alternativo (Isbister; Flanagan; Hash, 2010) e também como ferramentas de apoio e motivação (Leea et al, 2004). O sucesso dessas aplicações, entretanto, depende de algumas condições que não são de obtenção evidente.

Um videogame é um produto complexo, tanto do ponto de vista de engenharia de software quanto no que diz respeito ao caráter de instrumento pedagógico.

A construção de qualquer sistema computacional emprega técnicas e métodos da engenharia de software, uma área de conhecimento madura e que trata rotineiramente de requisitos oriundos de outros campos de saber, como mecânica, química ou psicologia. Entretanto, os jogos de computador contêm exigências que não fazem parte dos requisitos de sistemas comuns, como comerciais e de escritório. Sendo um nicho mais estreito e especializado, o desenvolvimento de videogames conta com um número menor de profissionais capacitados e/ou com experiência.

Já do ponto de vista pedagógico, existe um amplo arsenal de conhecimento desenvolvido em décadas de pesquisa, que deve ser trazido para a concepção de atividades e materiais que serão incorporados dentro de programas. Existem, todavia, aspectos peculiares dos videogames que tornam essa tarefa mais difícil. A interação entre uma pessoa e um computador se faz usando meios que existem há poucas décadas: teclados, mouses e monitores, o que é pouco tempo ao comparar com a tradição milenar do uso de papel, lápis, maquetes e outros materiais. Nessa escala de tempo faz sentido chamar o computador de um recurso "novo"; embora várias respostas e diretrizes já tenham sido encontradas, esse conhecimento é relativamente recente e não está popularizado. Eventualmente no futuro a ergonomia de interfaces se torne uma disciplina em cursos de licenciatura.

Algumas consequências das dificuldades elencadas se traduzem em implementações de jogos computacionais muito pobres. Muitos se limitam ao formato de um questionário, o que é resultado de um projeto pedagógico deficiente ou míope - ao acreditar que a simples vestimenta dada pelo computador representa algum avanço. É rara a preocupação com aspectos artísticos e a contratação de músicos e ilustradores profissionais. Por fim, a aplicação de critérios de 
qualidade de software e de engenharia ainda não é universalmente percebida como instrumento de qualidade e redução de custos e problemas.

Em geral observa-se uma dificuldade em aproximar mais as áreas de engenharia e de pedagogia. Cabe pesar também a relativa escassez de publicações que tratem simultaneamente dos aspectos computacionais e pedagógicos no desenvolvimento de jogos educacionais (Dondlinger 2007). Isto contribui para que erros sejam repetidos e acertos não estejam devidamente documentados para que possam ser empregados em novos projetos.

O presente trabalho traz pistas sobre o problema, sendo desenvolvido no âmbito de um projeto de mestrado. Busca-se elencar os pontos mais sensíveis no projeto e implementação de um jogo pedagógico. Para isso parte-se de uma análise de referencial bibliográfico e de conclusões obtidas em trabalhos do grupo de pesquisas do qual os autores fazem parte.

\section{Entretenimento e aprendizagem}

A junção entre jogo e educação não é recente, mas a adição de tecnologia, de modo mais evidente, sim (Oblinger, 2004). Embora renomados pesquisadores como Papert já trabalhassem com a idéia há muito tempo, os custos e a própria limitação tecnológica da época restringia os trabalhos práticos.

Os jogos representam uma forma de entretenimento universal, difundidos em diversas classes sociais e disponíveis para diversas faixas etárias (Brougère, 1995). A palavra 'jogo' é polissêmica em vários idiomas; em português, francês, inglês e espanhol, pode traduzir desde ideias leves como passatempo, brincadeira ou divertimento, até situações de rivalidade e competição.

Objetos e equipamentos são acrescentados a jogos há milênios e os computadores fizeram parte dessa história já na década de 60. Os videogames têm acompanhado a popularização do uso dos computadores e da Internet e se tornaram acessíveis a vários públicos. A indústria passou a realizar investimentos pesados em pesquisa e desenvolvimento, a tal ponto que as GPUs (unidades de processamento gráfico), originalmente concebidas para renderização gráfica, passaram a oferecer capacidade de processamento atrativa para aplicações sofisticadas de engenharia (Crow, 2004).

Em outra vertente, a aplicação de computadores na educação foi largamente estudada por Papert (1980). Seus trabalhos apontaram estratégias em que os computadores e a forma como interagimos com eles permitem criar situações prazerosas de descobrimento e construção de conhecimento. Os micromundos, um dos conceitos importantes explorados por Papert, podem desempenhar importante papel no aprendizado e na formação de conhecimento (Rieder e Brancher, 2002). Em síntese um micromundo é um espaço com elementos e regras próprias, que 
ditam o que é válido ou possível fazer. Para ilustrar, um jogo como "banco imobiliário" é um micromundo em que se compra e vende objetos, mas não que são transformados por operações como composição ou encaixe. Isso significa que nesse universo simulado há um conjunto limitado de operações possíveis. Essa limitação permite garantir o controle sobre as tarefas que serão realizadas dentro do micromundo: os participantes na atividade têm menores chances de fugir muito de um script pré-estabelecido e assim criar situações sem saída, que poderiam gerar frustração ou outros resultados negativos para ensino. Por outro lado, um conjunto muito pequeno de possibilidades tornaria a experiência desinteressante e engessada. O ambiente LOGO concebido por Papert (op. cit.) é um exemplo de implementação computacional de micromundo, em que se faz exploração livre e criativa, ou open-ended ${ }^{1}$. Comparativamente, videogames têm um compromisso mais claro com entretenimento e geralmente estabelecem regras que levam o utilizador à conclusão de um ou mais objetivos específicos.

Jogos determinam metas e exigem o exercício de habilidades para atingi-las. O senso de propósito e o prazer em atingir um nível de desempenho são ingredientes indispensáveis para que as pessoas se envolvam profundamente na atividade, a ponto de desconectarem da realidade. Esse fenômeno foi investigado por Csikszentmihályi (1990), que elencou situações propícias, elementos de antropologia, psicologia e fatores que, reunidos, podem desencadear essa reação por ele chamada de Flow State (estado de fluxo).

Há uma grande diversidade de formatos de videogames, resultado da ampliação do mercado; cada tipo pode atrair ou repelir diferentes jogadores. Títulos famosos que criam uma verdadeira legião de fãs ainda são objeto de críticas ou são completamente descartados por certos grupos. Isto deixa claro uma das peculiaridades sobre essas ferramentas: não se pode esperar que causem um efeito unânime. Um objetivo razoável é desenvolvê-los procurando torná-los agradáveis para a maioria dos usuários de um determinado segmento (Bethke, 2003), usando para isso critérios oriundos de áreas como ergonomia e conclusões obtidas em anos de desenvolvimento de videogames pela indústria. Uma vez vencidas as barreiras fundamentais de qualidade, tanto ergonômica quanto pedagógica, esses aplicativos tendem a tornar o aprendizado mais fácil, agradável e interessante (Rosas, 2003; Papastergiou, 2009).

Explorar jogos para finalidades diferentes do simples entretenimento levou ao conceito de Serious Games, ou Jogos Sérios (Stapleton, 2004; Zida, 2005) e a projetos como o “Serious Games Initiative" $^{\prime 2}$ que data de 2002. Um termo anterior é Edutainment, fusão de education e entertainment e que busca expressar a junção de ludicidade, prazer e, de outro lado, o ensino e

${ }^{1}$ Sem objetivo pré-determinado no início da atividade.

2 Projeto criado nos Estados Unidos; www.seriousgameinitiative.org. 
aprendizagem, estendendo-se a ideias tão diferentes como usar desenhos animados ou TV para veicular conteúdo (Rey-López, Fernández-Vilas, Díaz-Redondo, 2006).

Um exemplo relevante de Jogo Sério bem sucedido é o Americas' Army. Originalmente foi disponibilizado pelo exército americano com a finalidade de atrair recrutas e aproximar os jogadores da realidade do exército (Nieborg, 2004). Outra classificação estudada recentemente é de jogos chamados epistêmicos (Shaffer; Gee, 2006). Estes jogos simulariam situações encontradas em profissões e atividades específicas e são adequados para uso, por exemplo, no ensino baseado em problemas; um exemplo relevante nessa linha seria o desafio Sebrae (Lacruz, 2004). A filosofia de operação de tais jogos é considerada bem adaptada à formação atual esperada pelo mercado de trabalho (Ward 2002).

As fronteiras entre jogos educativos, jogos sérios e epistêmicos na literatura são imprecisas. $\mathrm{O}$ traço comum a todos é inserir objetivos pedagógicos dentro de uma atividade prazeirosa. Sem se ater a alguma tipologia é possível listar exemplos em inúmeros domínios, tão diferentes como a programação de computadores (Muratet et al., 2009) ou o ensino de línguas (Johnson, Vilhjalmsson, Marsella 2005). Outra característica é a simulação, intrínseca a muitos jogos (Wilson, 2008). Ela deriva em parte da maneira como esses programas funcionam internamente, geralmente usando uma arquitetura voltada a eventos discretos: o software opera internamente saltando entre estados bem definidos.

Em contraposição ao potencial favorável, nem todos jogos atingem seus objetivos. Alguns não tem sucesso como meio de entretenimento; não levam ao engajamento ou apresentam qualidade inferior à expectativa dos usuários, a ponto de não conseguir despertar o interesse. No domínio dos jogos para ensino, o aspecto de diversão muitas vezes não é tratado com a devida ênfase; por exemplo, os cenários não são atrativos, ou a 'jogabilidade' deixa a desejar - vide por exemplo (Elliott, Adams, Bruckman 2002). Outros títulos, embora divertidos, não atingem objetivos pedagógicos ou trazem uma contribuição de aprendizagem muito tímida, não justificando o uso.

Acertar o alvo duplo de entreter e ensinar é uma tarefa não trivial, mas existem alguns critérios e estratégias que podem ser aplicados de maneira consciente para potencializar as chances de sucesso.

\section{Implementação de jogos de computador}

Determinar quais as características dos Jogos Eletrônicos levam ao sucesso ou ao fracasso é uma tarefa quase tão difícil quanto seu desenvolvimento. Há videogames que funcionam extremamente bem apesar de um aspecto simples, sem a qualidade gráfica ou sonora sofisticada 
de produções comerciais milionárias (Papastergiou, 2009). Em outro extremo, existem softwares que terminam sendo enfadonhos apesar de todo investimento em sua construção (Bethke 2003).

Os motivos exatos que fazem alguns falharem e outros obterem sucesso ainda não estão claros. No caso de publicações sobre jogos para ensino, o foco recai mais na perspectiva educacional do que no projeto do software (Isbister; Flanagan; Hash, 2010). Já identificam-se indícios do que se deve realizar, mas são raras as metodologias para desenvolvimento. A escassez de publicações a respeito causa uma repetição de erros em vários projetos.

A criação de qualquer programa de computador pode tirar proveito de técnicas consagradas ao longo de décadas de experimentação e pesquisa. A engenharia de software lida com todos os problemas relacionados à construção e uso de programas, englobando o chamado ciclo de vida de software que se estende desde a concepção inicial até a retirada de funcionamento, passando pelo projeto, implementação, testes, uso e modificações. As técnicas usadas respondem em grande parte pelo sucesso ou fracasso de um produto (Pressman, 2001). Todo o processo envolve profissionais de diversas áreas, de acordo com os objetivos (Johnson; Schleyer, 2003). Vale lembrar que a indústria de jogos equipara-se atualmente à de cinema: os títulos de maior sucesso contam com orçamentos de milhões de reais. Alcançar uma qualidade similar a partir de uma abordagem amadora, ou "de garagem" conforme o jargão de Informática, parece um objetivo utópico.

Todo projeto deve partir de um planejamento, que será tão mais detalhado quanto mais complexo o produto ou maior o orçamento envolvido. Certas experiências de desenvolvimento em escolas ou grupos de estudo lançam mão de recursos baratos, mas que colocam em xeque os resultados; por exemplo, aproveita-se a habilidade de algum membro da equipe para desenhar cenários. Outra situação comum é implementar uma ideia e desenvolvê-la à medida em que o software é codificado. Em contraste, em uma abordagem profissional os programadores retardam a codificação até conhecer melhor o resultado final desejado. Existem modelos organizacionais corretos segundo o tamanho da equipe envolvida. Projetos pequenos podem se beneficiar de técnicas de menor custo, como XP ou SCRUM (Koscianski e Soares, 2006). 


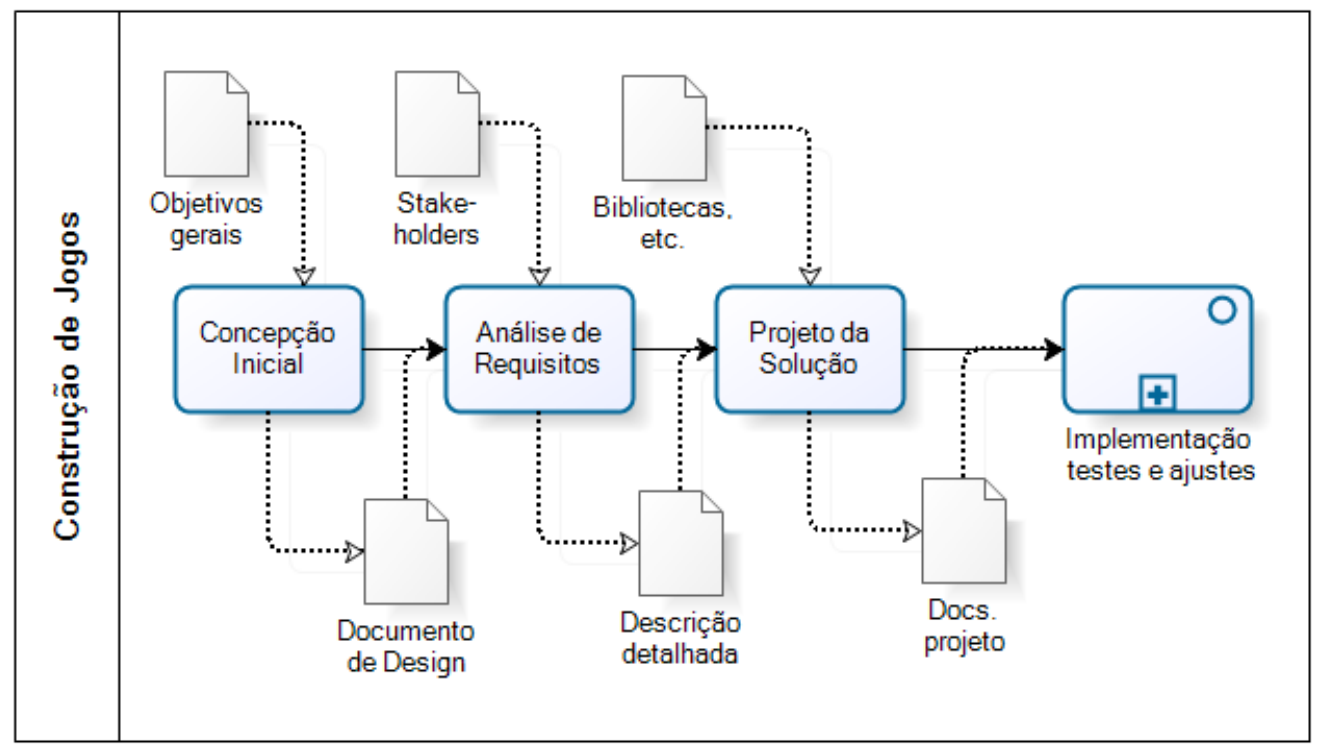

Figura 1 - processo aproximado de criação de um jogo computacional.

A Figura 1 ilustra de modo aproximado o processo de construção de um jogo; para conhecer mais a respeito, sugere-se consultar Flynt e Salem (2004) que tratam especificamente de jogos; ou Pressman (2001) e Sommerville (2007), que abordam engenharia de software de uma forma mais ampla.

A figura inicia com a concepção por meio de um documento de design ${ }^{1}$ de jogo; trata-se de uma descrição tão livre quanto possível do software, destinada a apresentar a ideia geral do produto sem se ater a aspectos de ordem técnica ou financeira. Ele serve como base para que toda a equipe de projeto discuta e defina, em uma etapa seguinte, os contornos do software que se pretende construir. Durante a elaboração desse documento são considerados diferentes gêneros, como 'corrida' e 'tiro ao alvo', opções de cenário e preferências do público-alvo. No caso de jogos educativos, os conteúdos e objetivos de aprendizagem também devem ser discutidos; algumas diretrizes a respeito serão discutidas em outra seção deste texto.

Durante a concepção um ponto muito delicado é o balanceamento entre conteúdo e diversão (Hayes, 2008). Ao deixar o conhecimento em segundo plano, ou tratá-lo inadequadamente, certos produtos apresentam desde falhas de ergonomia até a veiculação de informações erradas. Inversamente, há casos onde a ênfase em volume de conteúdo deixa em segundo plano o potencial do jogo. É muito comum encontrar softwares que implementam um simples questionário eletrônico, disfarçado em ações em que exprimem apenas sim/não, ao responder correta ou erroneamente. Exemplos são:

1 Preferiu-se aqui o anglicismo "design" para acentuar a diferença em relação ao projeto arquitetural.

R. B. E. C. T., vol 4, núm 1, jan./abr. $2011 \quad$ ISSN - 1982-873X 
- escolher objetos contendo respostas;

- mover objetos para posições pré-determinadas;

- alvejar objetos, fazendo mira com teclado ou mouse.

A análise de requisitos detalhará a visão geral traçada no documento de design. Nesse instante é preciso avaliar a influência de pessoas e organizações envolvidas na construção do produto, coletivamente conhecidas como stakeholders. Num jogo educacional deve-se ouvir, por exemplo, programadores, professores, especialistas em interfaces, desenhistas e alunos. Ponderando o peso das diferentes opiniões deve-se chegar a uma descrição do funcionamento, aparência, desempenho e outras características que identificam de forma unívoca o software e que atendam à demandas de cada pessoa que participa do projeto ou dele dependa de alguma forma.

A saída da etapa de projeto deve ser uma descrição da arquitetura do software. Ela mostra como implementá-lo usando ferramentas, bibliotecas de código pronto e outros recursos escolhidos pela equipe de desenvolvimento.

O último processo representado na figura agrupa a implementação e testes. Não necessariamente o desenvolvimento de um software é linear, como apresentado; há metodologias em que as etapas são cíclicas e a implementação se dá por etapas. Os testes finais servem para verificar o funcionamento do jogo e para calibrar o nível de dificuldade. Testes e revisões devem ser realizadas ao longo de todo o ciclo de vida, para reduzir a chance do produto desenvolvido apresentar diferenças se comparado ao que foi projetado. Esse problema é especialmente comum e difícil de tratar (Pressman 2001).

Isbister, Flanagan e Hash (2010) enumeram uma lista de considerações com um cuidado especial para três itens. Primeiro e nada trivial, garantir o divertimento no jogo; este é um requisito não funcional que deve fazer parte do projeto (Pluies, 2004). Segundo, devem haver critérios claros de qualidade, incluindo explicitamente a ergonomia (Röcker, Haar 2006); erros ou descuidos com os processos de engenharia de software podem por em risco todo projeto. 0 terceiro e certamente mais difícil, é fazer com que o ato de jogar em si já faça parte da aprendizagem. Essa ideia faz parte da filosofia dos jogos ditos 'epistêmicos' comentados anteriormente.

Alguns pesquisadores encaram o entretenimento como um componente tão importante quanto o conteúdo (Prensky, 2001b; Bellotti et all., 2009). O desenrolar do jogo deve fazer com que o usuário se sinta completamente imerso na atividade. Se o conteúdo a ser aprendido estiver completamente associado ao mecanismo do jogo, isto é, dentro da forma e das regras do ato de jogar, a informação poderá ser absorvida naturalmente, sem ser explicitada como um objetivo 
imposto. Essa sensação de continuidade de objetivos seria a situação ideal de acoplamento entre educação e entretenimento (Fisch 2005). Isso depende de elementos como roteiros, cenários, personagens e trilha sonora. A motivação é uma peça-chave para que o estudante esteja engajado no processo de ensino e aprendizagem e não apenas nas atividades de um jogo (Guillaume, Jouvelot, 2005).

A dificuldade para realizar tarefas - como atingir um alvo ou evitar inimigos - também afeta a motivação; ela deve ser tal que o usuário não seja nem frustrado, nem termine por perder o interesse frente a objetivos muito fáceis (Csikszentmihályi, 1990). Isso exige calibrar essa característica com muita precisão (Bethke, 2003). O objetivo é garantir que o usuário se sinta desafiado, mas dentro de um nível de conforto:

... watching kids work at mastering games confirms what I know from my own experience: learning is essentially hard; it happens best when one is deeply engaged in hard and challenging activities ${ }^{1}$ (Papert, 1998).

Alguns parâmetros que podem ser ajustados no controle de dificuldade são elencados na Tabela 1.

Tabela 1: Itens que podem afetar a dificuldade

\begin{tabular}{|l|l|}
\hline Parâmetro & Observações \\
\hline Limites de tempo & $\begin{array}{l}\text { Permite desafiar um jogador, ou simular a duração de } \\
\text { um fenômeno físico. Fácil de ajustar. }\end{array}$ \\
\hline $\begin{array}{l}\text { Precisão de adversários } \\
\text { controlados pelo } \\
\text { computador }\end{array}$ & $\begin{array}{l}\text { Pode ser implementado usando regras probabilísticas } \\
\text { de acerto/erro. Exige vários ciclos de teste. }\end{array}$ \\
\hline $\begin{array}{l}\text { Número, tamanho e } \\
\text { velocidade de obstáculos. }\end{array}$ & $\begin{array}{l}\text { Facilmente perceptível pelos usuários. Bastante difícil } \\
\text { de ajustar. }\end{array}$ \\
\hline $\begin{array}{l}\text { Número de vidas, "power- } \\
\text { ups" e munição }\end{array}$ & $\begin{array}{l}\text { O ajuste desses parâmetros pode exigir repassar o jogo } \\
\text { todo. Uma opção para facilitar o processo é definir o } \\
\text { estoque de itens na entrada de cada fase. }\end{array}$ \\
\hline
\end{tabular}

1 ...observando crianças se esforçando para dominar jogos confirma o que sei de minha própria experiência: o aprendizado é essencialmente difícil; ele acontece melhor quando alguém está profundamente engajado em atividades difíceis e desafiadoras. 
Um campo de pesquisa interessante diz respeito ao ajuste de dificuldade automático, em função do desempenho do jogador. Uma ideia semelhante já existe em sistemas tutoriais e em hipermídia adaptativa, em que o conteúdo apresentado ao usuário é modificado à medida em que o usuário interage com o sistema (Ahanger, Little 1997). A trilha musical e os efeitos sonoros também tem um impacto profundo na ambientação do jogador, podendo até afetar o nível de stress (Hébert et al. 2005).

\section{Bases pedagógicas no design de jogos}

A combinação eficaz entre prazer e aprendizado em um jogo começa no que se poderia chamar de estrutura pedagógica do software. Ela inclui elementos de design instrucional e de concepções sobre ensino aprendizagem (Polonoli, 2000). Um videogame pode ser usado como coadjuvante em sala, como ferramenta para trabalhar a afetividade da classe, ou então como um veículo independente de conteúdo.

Dentro do contexto de aprendizado sugere-se que há duas fontes de motivação: a baseada em perícia e a baseada em desempenho (Meece, Andreman \& Anderman, 2006). No primeiro caso o indivíduo está determinado a vencer um obstáculo e obtem prazer por conseguir cumprir uma tarefa. No segundo, a satisfação é obtida quando se atinge um dado desempenho em uma escala, que pode envolver medidas como velocidade, distância ou número de sub-objetivos alcançados. Ambas visões são contempladas pela teoria do fluxo (Csikszentmihályi, 1990). Ames (1992) baseou-se em estudos sobre cognição e comportamento para elencar critérios que o professor pode usar em sala:

1. tarefas: existe mais preferência por variedade e diversidade. Uma atividade repetitiva tem menores chances de trazer interesse. Um claro senso de propósito permite que os estudantes se envolvam naturalmente em uma atividade. A interação com a tarefa, com professor e colegas pode influenciar na percepção dos objetivos e sua variabilidade.

2. avaliação e reconhecimento: a forma de avaliação e o recebimento de recompensas tem efeitos claros. Estudantes tendem a ser sensíveis a como o próprio desempenho é avaliado e também como é visto dentro do grupo em que convivem. Se bem gerida, a competição por resultados pode ter um efeito salutar.

Esses dois pontos coincidem com critérios apresentados em (Qin, Rau e Salvendy 2010), a respeito do repertório de tarefas e da dificuldade crescente dentro de cada tarefa separada. 
O primeiro item - a variabilidade de tarefas - pode ser conseguido fazendo variar regras ao longo do jogo, introduzindo restrições que exigem mais atenção ou perícia. Também é possível manter as regras inalteradas mas mudar cenários, inimigos e cenários a cada fase. A curiosidade por descobrir o que surgirá na próxima cena faz com que os usuários se mantenham interessados.

Quanto ao segundo item - a avaliação e reconhecimento - a pontuação é o mecanismo natural para implementação dentro de um jogo. É interessante notar como é comum professores criarem jogos com pontuação dentro da classe; exemplos são cartazes com rankings dos melhores leitores, ou tabelas com estrelas para os estudantes com mais tarefas bem realizadas. Esses objetivos externos ao aprendizado em si, quando apresentados e geridos corretamente, proporcionam um clima agradável de realização. Por exemplo, o professor pode designar um objetivo básico, como "ganhar 3 estrelas" factível para todos os indivíduos da classe e trabalhar o sentimento de satisfação de cada aluno que atinge essa marca. Regras envolvendo respeito aos colegas e ajuda mútua também podem ser estabelecidas para criar um código de ética. Isso permitirá dosar a competição entre alunos e evitar derrapagens como comparações negativas.

As regras de pontuação em um videogame podem somar tanto objetivos educacionais quanto os resultados ligados à habilidade de jogar em si. Algumas maneiras de fazer isso são (Torrente et al. 2008): associar notas com o alcance de certos estados no jogo (como acabar uma fase); incrementar nota à medida em que etapas são vencidas; ou aumentar a pontuação em função da velocidade com que as tarefas são cumpridas. Essa mescla ajuda a aproximar "jogo" e "ensino" dentro da percepção do usuário do software.

A escolha de gênero, como plataforma ou corrida, depende tanto de um perfil do grupo de usuários, quanto das oportunidades para mesclar conteúdos. Uma das pistas para tratar essa questão é fazer um mapeamento entre conteúdos pedagógicos e elementos de jogos, tais como estilos, regras e objetivos; a partir daí deriva-se um conjunto de cenários potenciais para posterior refinamento e implementação (Koscianski, 2010). Usando a mesma ideia-base, como tiro ao alvo, pode-se variar cenários, inimigos e sons ao longo do jogo de forma a conseguir resultados completamente diferentes. O storyboard é uma ferramenta valiosa para esboçar conceitos e discuti-los dentro de uma equipe de projeto de jogos.

A interface de um videogame é possivelmente o componente mais delicado em um produto educacional. As regras internas de funcionamento são sentidas ao longo das atividades mas têm um impacto menos imediato no utilizador, envolvendo assim menos sua afetividade e a aceitação do software. Ao projetar a interface deve-se ter em mente um projeto instrucional, avaliando-o com tanto ou mais cuidado como o dispensado a uma aula tradicional. Modelos como o de Robert Gagné podem ajudar a organizar a sequência de atividades e as interações a serem programadas (Blunt, 2006), ou estabelecer uma lista de verificação a ser obedecida. Por exemplo: 
- guiar o aluno na realização das tarefas; por exemplo provendo explicações mais detalhadas no caso de erros;

- revisar conhecimentos anteriores; por exemplo, um jogo pode começar com alguns problemas fáceis antes de incluir informação que se considera nova para o estudante;

- manter a atenção; aumentar a dificuldade de maneira crescente e incluir situações inesperadas nas regras e no cenário evitam a sensação de monotonia.

Diretrizes clássicas de ergonomia são de extrema valia no projeto. Elas incluem, por exemplo, o tratamento de cores, distribuição de objetos e uso adequado de textos, controles e fluxo de tarefas. Há uma literatura extensa nessa área e heurísticas de avaliação de interface diretamente aplicáveis a jogos.

A construção "interna" do jogo, no que se refere à bases de dados e navegação pelos conteúdos e problemas, deve buscar alicerce em teorias de ensino-aprendizagem. Como o universo dos jogos abre a possibilidade de criar regras livremente, o desenvolvedor de um produto educacional tem margem para opções como o jogo individual, a disputa entre duas ou mais pessoas ou ainda a aprendizagem cooperativa (Ke, 2006); dentro de cada uma a disposição de informações e problemas pode ser variada com muita liberdade. Um caminho para fazer a ligação entre regras de jogo e conteúdo didático é apoiar-se em verbos de ação que descrevam esses dois universos (Koscianski 2010). A partir da identificação de ações similares é possível propor traduções de conteúdo a estudar para um jogo. Essa estratégia funciona especialmente bem quando o conteúdo possuir algum aspecto dinâmico; exemplo disso são processos químicos ou biológicos que o estudante deve compreender e memorizar.

A abordagem mais comum em videogames ainda é a comportamentalista ou de estímuloresposta. Geralmente é construído um catálogo de perguntas ou problemas com as respectivas respostas, para que o software as apresente em sequência ou em sorteio dentro de níveis de dificuldade. Criar um sistema de instrução programada, mesclando novos conteúdos e problemas também é relativamente fácil tanto do ponto de vista de preparar a sequência de informações e perguntas, quanto de criar um jogo em si. Isso pode explicar porque o estilo pergunta-resposta é predominante. Entretanto, também se encontram softwares que seguem uma linha construtivista ou a construcionista (Dondlinger, 2007; Wilson, 2008), nos quais as tarefas não são tão lineares. Uma discussão interessante a esse respeito é apresentada por Juul (2007). Para aumentar a flexibilidade de jogos há gêneros como o RPG (role-playing-game) ou de simulação (Wilson, 2008), que oferecem a possibilidade de criar objetivos mais complexos mas que, ao mesmo tempo, exigem um esforço bem maior de implementação. Um exemplo clássico nessa linha é TIM, The 
Incredible Machine; o jogo permitia combinar livremente objetos para criar um mecanismo que devia desempenhar uma tarefa, como lançar bolas em uma caixa, apagar uma vela ou abrir uma gaiola.

A Figura 2 a seguir sintetiza essas ideias.

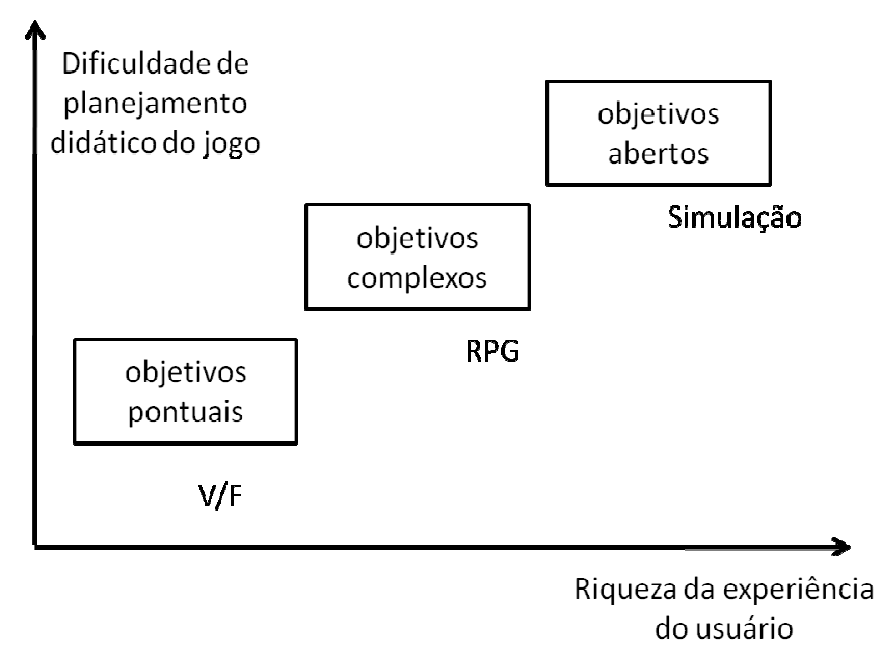

Figura 2 - estilos de jogos

Seguindo a Figura 2, pode-se pensar em uma classificação de jogos em três níveis, segundo as tarefas que eles propõem aos estudantes:

objetivos pontuais: correspondem ao formato de questionário ou de perguntas fechadas. Podem ser implementados usando gêneros como tiro-ao-alvo e de ação em geral, usando perpectivas em primeira e terceira pessoa;

objetivos complexos: jogos nessa categoria permitem criar objetivos fracionados em etapas que devem ser cumpridas em ordem ou não. Os RPG (role-playing-game) e os adventures são os melhores exemplos. O jogador pode precisar encontrar e combinar objetos, ou resolver problemas em que cada resposta é usada como entrada em uma nova situação;

objetivos abertos: dificilmente um jogo será completamente desprovido de objetivos; os jogos mais flexíveis estabelecem, no mínimo, um regime de pontuação que permite ao usuário decidir por uma linha de ação. Um exemplo clássico é SimCity.

Conforme a descrição de Moreira (2008), a aprendizagem ocorre quando um novo conhecimento é relacionado aos conhecimentos já adquiridos previamente. Esse princípio vem da teoria da Aprendizagem Significativa de Ausubel (2000) e também pode ser utilizado para organizar a disposição de conteúdos no software.

Aprender significativamente se traduz em interiorizar o conhecimento relacionando-o a um ou mais conhecimentos prévios. Jogos são apontados como possíveis instrumentos de 
aprendizagem significativa (Rosas et all, 2003) e podem servir, por exemplo, como organizadores prévios. Nesse caso o jogo pode apresentar situações que ilustram problemas que, mais tarde, serão abordados em mais detalhe pelo professor. Para considerar um exemplo, o software pode requerer a manipulação de objetos usando ângulos e forças para vencer obstáculos dentro de um campo gravitacional ou eletromagnético. Mais tarde em sala de aula esse conteúdo pode ser retomado em bases teóricas, motivando conceitos como momento e inércia e uma representação abstrata usando matemática.

Jogos computacionais comportam a simulação de mecanismos de complexidade arbitrária. Esses mecanismos podem retratar conceitos abstratos ou fenômenos não visíveis ou não acessíveis, como por exemplo processos físicos ou químicos em escala molecular. Pode-se elaborar metáforas para reificar conceitos abstratos em jogos (Koscianski, 2010); estas podem servir como organizadores prévios para o material examinado posteriormente em sala.

\section{Conclusões}

Trabalhar com jogos eletrônicos na educação não é uma tarefa simples e exige muitos recursos. Ainda parece distante o objetivo de criar uma metodologia que integre os problemas de diversão e educação de uma maneira que se possa chamar de correta. No entanto, há considerações norteadoras que apontam uma convergência dos pesquisadores em torno dos requisitos para implementar um software desse tipo.

As características de entretenimento e informação são necessárias para o efeito desejado de engajamento no aprendizado. Criar roteiros, cenários e situações interessantes ainda é uma tarefa que depende de talento pessoal; contudo, é possível seguir regras de ergonomia e listas de verificação de critérios, de maneira a evitar resultados puramente aleatórios.

A construção de jogos computacionais demanda recursos tecnológicos e financeiros. A implementação necessita de profissionais de diversas áreas e de materiais adequados para atingir um nível de qualidade de acordo com a expectativa do público-alvo. É importante lembrar que, geralmente, este público vêm crescendo diante de jogos das grandes produtoras, que são tomados como base para avaliação dos jogos educacionais. Assim sendo, um título educativo com um mínimo de ambição deve prever, por exemplo, investimentos com equipes de artistas gráficos e músicos e não dar margem a improvisações. Tanto o tratamento de engenharia, quanto o pedagógico, merecem igual atenção.

O aprendizado deve ser implícito tanto quanto possível; o fundamento de um jogo eletrônico não é exatamente compatível com a ideia de uma apostila digital. Ao contrário, o 
software tem por princípio o divertimento. Alinhar a isto os objetivos educacionais permite que o estudante jogue por prazer e não tenha a percepção de conteúdos pedagógicos como elementos estranhos à essa atividade. Determinar em que medida essa mescla é possível e, em particular, como pode ser sistematizada, são perguntas que permanecem em aberto. Felizmente existem algumas diretrizes aplicáveis, como trabalhar conteúdos de aprendizado dentro das regras do jogo, replicar situações reais usando metáforas ou ainda relacionar mecanismos a estudar com mecanismos do micromundo de um jogo.

Finalmente, a adoção de uma Teoria de Aprendizagem para embasar a construção de jogos educacionais é imprescindível e não deve ser relegada a segundo plano. Ela não apenas evitará erros na elaboração de material que veiculará conteúdo, como textos e gráficos, mas fornecerá subsídio para organizar toda a interface do software, passando pelas regras do jogo até a sequência de atividades oferecida ao usuário. Idealmente um software educativo seria acompanhado de um manual de aplicação pedagógica, tornando clara sua concepção e como seu uso foi pensado em relação a uma sala de aula, aplicação em laboratório ou atividade isolada do estudante.

\section{Agradecimento}

Os autores agradecem à Fundação Araucária do Paraná pelo suporte financeiro ao projeto.

\section{Referências}

Ahanger, G. , Little, T. D. C. Easy Ed: An Integration of Technologies for Multimedia Education, in S. Lobodzinski and I. Tomek (Eds.) Proc. of WebNet'97, World Conference of the WWW, Internet and Intranet, pp. 15-20, Toronto, Canadá, 1997.

Ausubel, D. P. Aquisição e Retenção de Conhecimentos: Uma Perspectiva Cognitiva. Plátano Edições Técnicas. Lisboa, 2000.

Badilla-Saxe, E. Subversive Change: Empowering Educators to Lead Innovation from Within. Journal of Systemics, Cybernetics and Informatics, vol. 2, n. 2, 2004.

Bellotti, F.; Berta, R.; Gloria, A. D.; Primavera, L. Enhancing the educational value of video games. Comput. Entertain., v. 7, n. 2, p. 1-18. doi: 10.1145/1541895.1541903, 2009.

Bethke, E., Game Development and Production, Wordware Publishing, Inc., 2003.

Blunt, R. D. A causal-comparative exploration of the relationship between game-based learning and academic achievement: teaching management with video games. Tese de Doutorado. Walden University. USA. 2006. 
Brougère, G. Jeu et éducation. Paris: L'Harmattan, 1995.

Crow, T. S. Evolution of the Graphical Processing Unit. Dissertação de Mestrado. University of Nevada, USA, 2004. (http://www.cse.unr.edu/ fredh/papers/thesis/023-crow/GPUFinal.pdf).

CSikszentmihályi, M. Flow: the psychology of optimal experience. 1 ed. New York: Harper Perennial. 1990.

Dondlinger, M. J. , Educational Video Game Design: A Review of the Literature, Journal of Applied Educational Technology, Volume 4, Number 1 Spring/Summer 2007.

Dondlinger, M. J. Educational Video Game Design: A Review of the Literature. Journal of Applied Educational Technology. Vol 4., Num. 1, 2007.

Elliott, J. , Adams, L. , Bruckman, A.. No Magic Bullet: 3D Video Games in Education, In Proceedings of the International Conference of the Learning Sciences, ICLSICLS 2002; Seattle, Washington, October 2002.

Fisch, S. M. Making Educational Computer Games "Educational", in Proceedings of the $\mathbf{2 0 0 5}$ conference on Interaction design and children. Boulder, Colorado, USA, June 8-10, 2005.

Guillaume, D. Jouvelot, P. Motivation Driven Educational Game Design: Applying Best Practices to Music Education. In Advances in Computer Entertainment Technology (ACE), Valencia, Espanha. 2005.

Hayes, E. Game content creation and it proficiency: An exploratory study. Computers \& Education, v. 51, n. 1, p. 97-108. doi: 10.1016/j.compedu.2007.04.002, 2008.

Hébert, S., Béland, R., Dionne-Fournelle, O., Crête, M., Lupien, S. J.. Physiological stress response to video-game playing: the contribution of built-in music. Life Sciences, 76, 2005.

Isbister, K.; Flanagan, M.; Hash, C. Designing games for learning: insights from conversations with designers. In: Proceedings of the 28th international conference on Human factors in computing systems, p.2041-2044, Atlanta, Georgia, USA. 2010.

Johnson, W. L. , Vilhjalmsson, H. , Marsella, S.. Serious games for language learning: How much game, how much AI?, In Procedings of the International Conference on Artificial Intelligence in Education. Amsterdam: IOS Press. 2005.

Juul, J. , Without a goal. In Tanya Krzywinska and Barry Atkins (eds): Videogame/Player/Text. Manchester: Manchester University Press 2007.

Ke, F. Classroom goal structures for educational math game application. In: Proceedings of the 7th international conference on Learning sciences. Bloomington, Indiana. 2006. 
Koscianski, A., \& Soares, M. Qualidade de Software. São Paulo.: Editora Novatec. 2006.

Koscianski, A. Changing the rules: injecting content into computer games. In: Francisco V. C. Ficarra. (Org.). Quality and Communicability for Interactive Hypermedia Systems: Concepts and Practices for Design. Hershey, Pennsylvania, USA: IGI Global, v. , p. 160-174. 2010.

Lacruz, A. Jogos de Empresas: considerações teóricas. Caderno de pesquisa em Administração FEA - USP, vol. 11, n. 4, 2004.

Leea, J. , Luchinia, K. , Michaela, B. , Norrisb, C. , Solowaya, E. . More than Just Fun and Games: Assessing the Value of Educational Video Games in the Classroom. In Proceedings of humancomputer interaction, CHI 2004 Vienna, Austria. 2004.

Masini, E. F. S.; Moreira, M. A. Aprendizagem significativa. VETOR, 2008.

Meece, J. L. , Anderman, E. M., Anderman, L. H. , Classroom goal structure, student motivation, and academic achievement. Annu. Rev. Psychol. Vol 57, pp487-503. 2006.

Muratet, M.; Torguet, P.; Jessel, J.; Viallet, F. Towards a serious game to help students learn computer programming. Int. J. Comput. Games Technol., v. 2009, p. 1-12, 2009.

Nieborg, D. B. America's Army: more than a game. Transforming knowledge into action through gaming and simulation. Ed. \& Thomas Eberle Willy Christian Kriz. München: ISAGA (International Simulation \& Gaming Association) Conference Proceedings, 2004.

Oblinger, D. The next generation of educational engagement. Journal of Interactive Media in Education, v. 8, p. 1-18, 2004.

Papastergiou, M. Digital Game-Based Learning in high school Computer Science education: Impact on educational effectiveness and student motivation. Comput. Educ., v. 52, n. 1, p. 1-12, 2009.

Papert, S. 1998. Does Easy Do It? Children, Games, and Learning. Game Developer Magazine, June 1998.

Papert, S. Mindstorms: children, computers, and powerful ideas. Basic Books, Inc. New York, USA, 1980.

Pluies, J. L. Jeu, TIC et apprentissage. Dissertação de Mestrado. Université de Paris 3 - Sorbonne Nouvelle. 2004.

Polonoli, K.E., What makes educational software educational? Virginia Society for Technology in Education Journal, 15, 44-51, 2000.

Prensky, M. Digital Natives, Digital Immigrants, On the Horizon, Vol. 9, N. 5, NCB University Press, 2001a.

Prensky, M. Digital Game-Based Learning. McGraw-Hill. 2001b.

Pressman, R. S. Software Engineering: A Practitioner's Approach (6 ed.): McGraw-Hill. 2001

R. B. E. C. T., vol 4, núm 1, jan./abr. $2011 \quad$ ISSN - 1982-873X 
Qin, H., Rau, P.L P., Salvendy, G.: Effects of different scenarios of game difficulty on player immersion. Interacting with Computers. Vol 22., N. 3. 2010.

Rey-López, M. , Fernández-Vilas, A. , Díaz-Redondo, R. P.. A Model for Personalized Learning Through IDTV. Adaptive Hypermedia and Adaptive Web-Based Systems. Lecture Notes in Computer Science, Volume 4018, pp. 457-461, 2006.

Rieder, R.; Brancher, J.D. Development of a micro world for the education of the Fundamental Mathematics, using OpenGL and Delphi. In: Conferencia Latinoamericana de Informática, 28., 2002, Montevideo, Uruguay. Actas do X Congreso Iberoamericano de Educación Superior en Computación. Montevideo: infoUYclei, 2002. p. 162-173.

Röcker, C. Haar, M., Exploring the usability of video game heuristics for pervasive game development in smart home environments. In Proceedings of the Third International Workshop on Pervasive Gaming Applications - PerGames 2006, pages 199-206, Dublin, Ireland, 2006.

Rosas, R., Nussbaumb, M., Cumsillea, P., Marianovb, V., Correa, M., Floresa, P., Graua, V., Lagosa, F., Lopeza, X., Lopeza, V., Rodriguez, P., Salinas, M.. Beyond Nintendo: design and assessment of educational video games for first and second grade students. Computers \& Education 40. pp 7194. 2003.

Shaffer, D. W.; Gee, J. P. How Computer Games Help Children Learn. Palgrave Macmillan, 2006.

Sommerville, I., Engenharia de Software. Pearson Education do Brasil. 8. Edição, 2007.

Stapleton, A., Serious Games: Serious Opportunities. Australian Game Developers' Conference, Academic Summit, Melbourne, VIC, 2004.

Torrente, J., Moreno-ger, P., Fernández-Manjón, B. , Sierra, J. L.. Instructor-oriented Authoring Tools for Educational Videogames, in Proceedings of ICALT '08. Eighth IEEE International Conference on In Advanced Learning Technologies, Santander, Spain, 2008, pp. 516-518.

Vrasidas, C. Issues of pedagogy and design in e-learning systems. In: Anais... . p.911-915. Nicosia, Cyprus: ACM. doi: 10.1145/967900.968086, 2004.

Ward, J. D. A Review of Problem-Based Learning. Journal of Family and Consumer Sciences Education, vol. 20, n. 1, 2002.

Wilson, K. A., Bedwell, W. L., Lazzara, E. H., Salas, E., Burke, C. S., Estock, J. L., Orvis, K. L., Conkey, C. Relationships Between Game Attributes and Learning Outcomes. Review and Research Proposals. Simulation \& Gaming. Vol. 20, N. 10. 2008. 
Leandro Augusto Kisielewicz. Faculdade Educacional de Ponta Grossa. Professor da Faculdade Educacional de Ponta Grossa. Graduação em Bacharelado em Informatica pela Universidade Estadual de Ponta Grossa. leandro.kisielewicz@gmail.com

André Koscianski. Universidade Tecnologica Federal do Paraná. Professor da graduação e mestrado PPGECT da Universidade Tecnologica Federal do Paraná. Doutor em Simulação - Institut National Des Sciences Appliquées de Rouen. koscianski@utfpr.edu.br 